\title{
Consensus building for the development of guidelines for recommending mobility service dogs for people with motor impairments
}

\author{
Claude Vincent ${ }^{\mathrm{a}, \mathrm{b}, *}$, Lise Poissant ${ }^{\mathrm{c}, \mathrm{d}}$, Dany H. Gagnon ${ }^{\mathrm{c}, \mathrm{d}}$, Hélène Corriveau ${ }^{\mathrm{e}}$ and \\ members of the ADMI group \\ ${ }^{a}$ Centre for Interdisciplinary Research in Rehabilitation and Social Integration, Institut de Réadaptation en \\ Déficience Physique de Québec, Quebec, Canada \\ ${ }^{\mathrm{b}}$ Department of Rehabilitation, Université Laval, Quebec, Canada \\ ${ }^{\mathrm{c}}$ Centre for Interdisciplinary Research in Rehabilitation of Greater Montreal, Institut de Réadaptation \\ Gingras-Lindsay-de-Montréal, Montreal, Canada \\ ${ }^{\mathrm{d}}$ School of Rehabilitation, Université de Montréal, Montreal, Canada \\ ${ }^{\text {e }}$ School of Rehabilitation, Université de Sherbrooke, Sherbrooke, Canada
}

\begin{abstract}
.
BACKGROUND: According to recent research, mobility service dogs (MSD) improve grasping ability, autonomy in ADL, manual wheelchair propulsion, walking, transfers, psychosocial aspects, reintegration into normal life, and satisfaction with important occupations, and decrease pain in manual wheelchair users' shoulders/wrists. However, it remains a challenge for rehabilitation professionals to recommend MSD for different profiles of neurological disorders.

OBJECTIVE: Formulate guidelines to support the decision-making process of rehabilitation professionals recommending MSD. METHODS: Focus groups with MSD experts ( 7 therapists, 4 trainers, 3 managers, 5 users) responded to four research questions. They had to formulate and prioritize criteria to inform the recommendation of MSD for three clinical cases: A-tetraplegia with powered wheelchair, B-paraplegia with manual wheelchair, and C-ambulatory (incomplete SCI or neurodegenerative disease).

RESULTS: For the decision-making process of recommending MSD, six main variables were identified: scientific evidence cited (they are different among clinical cases), added value of MSD compared to other assistive devices (dissimilar among clinical cases), prioritization of personal (7), environmental (8) and canine (6) characteristics, and possible negative consequences in MSD user's life (stigmatization, resilience, care burden, authority or obedience).

CONCLUSIONS: The results provide the basis for the development of clinical practice guidelines for occupational therapists and physiotherapists recommending MSD to individuals presenting various profiles of neurological disorders.
\end{abstract}

Keywords: Service dog, assistant dog, assistive device, spinal cord injuries, rehabilitation, wheelchair

\section{Introduction}

Mobility service dogs (MSD) compensate for motor impairments and functional limitations. They can

\footnotetext{
*Corresponding author: Claude Vincent, Department of Rehabilitation, Université Laval, Pavillon Vandry, 1050 av. de la Médecine, Quebec City, G1V 0A6, Canada. Tel.: +1 4186562131 \# 6078; Fax: +1 418656 5476; E-mail: claude.vincent@rea.ulaval.ca.
}

help with standing balance, walking, grasping, pulling a manual wheelchair and access to the environment for powered wheelchair users. In Quebec, three thousand MSD have been assigned between 2003 and 2015. It is estimated that 310 MSD were in use in 2015 (N. Champagne, personal communication, November 26, 2015). In Quebec, the large majority of MSD are trained by the MIRA Foundation, a non-profit organization selffinanced through sponsorships and donations. To get a

ISSN 1055-4181/16/\$35.00 (c) 2016 - IOS Press and the authors. All rights reserved

This article is published online with Open Access and distributed under the terms of the Creative Commons Attribution Non-Commercial License (CC BY-NC 4.0). 
MSD, people with motor impairments and documented functional limitations may apply to the MIRA Foundation using the application form on its Website [1]. If deemed eligible based on the various assessment criteria (e.g., health and disability questions, family situation, dwelling, lifestyle, education/employment situation), they must stay at the training centre for 18 days for partnering and training with a prequalified twoyear-old dog to learn new skills. Then, over the next four months, they must use their dog as an assistive device in their community to continue optimizing its use in real life. While it costs the MIRA Foundation $\$ 30,000 \mathrm{CAD}$ on average to train a dog, all are supplied free of charge to selected users. In Quebec, after six months, users can apply to be assessed by an occupational therapist or physiotherapist to see if they qualify for a program reimbursing expenses related to the use of a MSD [2]. In addition to an initial reimbursement of $\$ 210$ CAD upon acceptance into the program, MSD users receive $\$ 1028$ CAD per year to cover other types of expenses (e.g., food, grooming and veterinary care).

Despite growing evidence of the clinical efficacy and positive impacts of MSD [3-10], the decisionmaking process for recommending a MSD is not optimal with respect to clinical knowledge. Through a literature review on the subject of clinical guidelines for recommending MSD, if those exist in other countries, no publications have detailed or mentioned them. What we found concern animal assisted therapy (e.g. autism, youth, mental health, psychotherapy, and socialisation) rather than 'animal assisted technology' that is the purpose of our paper. The most complete reference on the subject is the Handbook on animal assisted-therapy: theoretical foundations and guidelines for practice, edited by Aubrey H. Fine, in 2010 [11]. Unfortunately, there is no mention of any guideline validated for occupational therapists or physiotherapists working with people with motor impairments.

In other words, while it might seem relatively easy to evaluate the dog's impact on autonomy and other parameters, being taught when and how to recommend a dog for specific clients based on their profile remains challenging. Part of the problem lies in the fact that it is not occupational therapists or physiotherapists who assign the dogs. This creates a clinical misconception of the real usability of the dogs (i.e., efficacy, security, satisfaction and efficiency) [12,13], of the facilitators or barriers to recommending MSD, and of the added value of the MSD compared to other assistive devices. Currently, rehabilitation professionals have difficulty advising clients with regard to getting a dog because they do not have guidelines concerning the personal characteristics expected of clients who might benefit most from a MSD, the environments receptive to a MSD, and the specific characteristics of the MSD itself. According to Scherer [14], it is important to properly document all of these variables in the matching person and technology assessment process.

To address these gaps (i.e., scientific evidence, added value of having a dog, person-environmenttechnology matching process, and disadvantages of having a dog), we conducted a study with individuals who have complementary types of expertise with MSD to formulate guidelines to support the decision-making process of rehabilitation professionals involved in the process of recommending MSD. Specifically, considering the types of knowledge gaps, four research questions were formulated:

(1) What scientific evidence contributes to the decision-making process of recommending MSD?

(2) What is the perceived added value of MSD in comparison to other available assistive devices?

(3) What characteristics of the user, the environment or the MSD contribute to the decision-making process?

(4) What foreseeable negative consequences in the user's life linked to the use of a MSD might influence the recommendation process?

\section{Methods}

\subsection{Research design}

To address those research questions, we chose a design employing focus groups of experts (rehabilitation professionals, trainers, managers, users). An individual meeting was held with one of the participants who could not attend any of the focus groups. The study was approved by the research ethics board (CRIR-6330711). All participants signed a consent form.

\subsection{Population}

All rehabilitation professionals and managers approached for the study worked in four rehabilitation centres with clients who could potentially qualify for a MSD, and they all agreed to participate. All the dog trainers worked at the MIRA Foundation; the five trainers with the most expertise (out of 12) were recruited by the Foundation's management to participate. For the 
recruitment of MSD users, a convenience sample was formed in order to have different user profiles (e.g., walker, manual and powered wheelchair user). They were identified by a physiotherapist involved in the program for reimbursing expenses related to the use of a MSD; six users were invited to participate.

\subsection{Focus group sessions}

All of the focus groups sessions (between 3 and 6 people) were facilitated by two members of the research team (LP and CV). A guide for an interview lasting approximately two-and-a-half hours was developed based on the usual steps in planning a focus group [15]. It was designed with the help of the Assistance Dog for Mobility Impairments (ADMI) group, which involves six researchers (see Acknowledgements). Each session followed the same eight-step sequence: 1) introduction; 2) visual presentation by the facilitator highlighting 10 statements summarizing the results of the research previously done by the ADMI group; 3) distribution and presentation of the teaching material to the participants, i.e. hard copy of the data presentation, small cards and clinical cases (see box 1); 4) prioritization of the research statements for each typical clinical case by each participant (box 1); 5) discussion and emergence of a consensus concern-

Box 1 - Teaching material

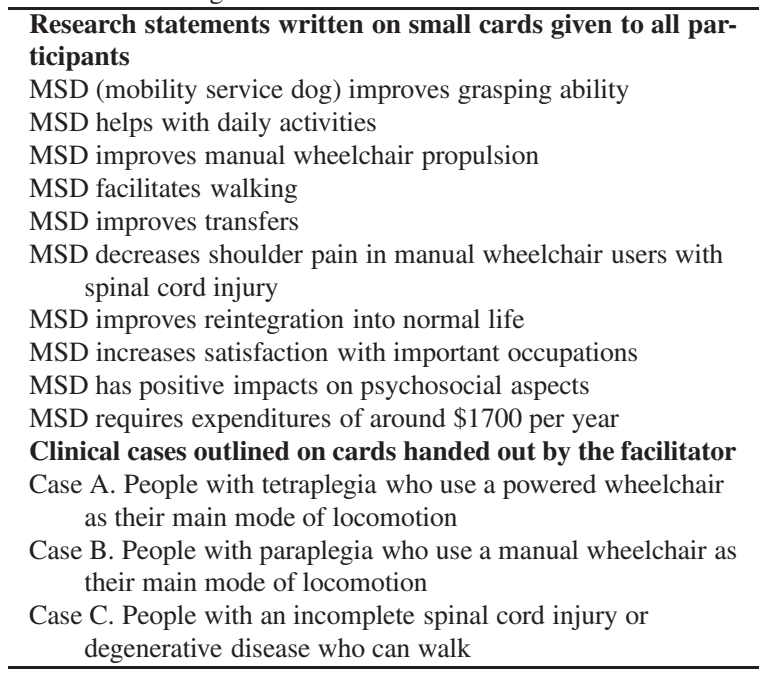

ing the five most important statements for each case; 6) discussion concerning the added value of MSD versus different assistive devices applicable to the three clinical cases (added value was defined in terms of efficacy (superior, inferior or equivalent to another assistive de- vice) and complementarity); 7) discussion concerning the characteristics of the MSD, of the user and of the environment that would lead to the recommendation of a MSD; and 8) discussion of the possible negative consequences in daily life of having a MSD.

\subsection{Data analysis}

The discussions were audiotaped. Concerning question 1, a quantitative approach was used. For each clinical case, weighting was added separately to each of the five key research statements identified as being the most important ( 5 points for the 1 st criterion down to 1 point for the 5 th in order of importance). A total score per criterion was calculated with a maximum possible score of 95 . The most frequent ranking assigned by the 19 participants was also reported for each statement. For the data analysis for questions 2, 3 and 4, an inductive and deductive thematic qualitative approach [16] was used. The audiotaped discussion was transcribed. The text was then coded using ten inductive themes directly linked to the expected answers to research questions 2, 3 and 4. Ten main themes/codes were used: MSD superior, inferior, equivalent or complementary; physical, human and organizational environment; characteristics of the MSD user; dog's skills; negative consequences of the MSD. Once the text was coded using these themes, the next step was to highlight the different aspects to be considered (i.e. deductive themes). For each of the deductive themes, a typical extract from the transcript was rephrased as an affirmative, interrogative or imperative sentence to create an understandable guideline for rehabilitation professionals. Detailed information on themes for questions 3 and 4, is synthesized in Tables 3 and 4. The coding step was followed by categorization, which involved proposing a "decision-making process diagram to recommend a mobility service dog". This was necessary to better highlight interrelationship between themes and deductive themes.

\section{Results}

There were 4 focus group sessions and 1 individual meeting for a total of 19 expert participants. There were two groups including the rehabilitation stakeholders (3 managers, 3 occupational therapists, and 4 physiotherapists), one group including the producers (4 dog trainers) and one group including the users (5 MSD users). The participants had diversified and complementary expertise as detailed in Table 1. 
Table 1

Focus group's participants and their expertise with mobility service dogs (MSD) $(n=19)$

\begin{tabular}{|c|c|}
\hline Rehabilitation professionals ( $n=7$ females) & MSD expertise \\
\hline $\begin{array}{l}2 \text { occupational therapists, city } 1[1] \\
1 \text { occupational therapist, city } 2^{[2]} \\
2 \text { physiotherapists, city } 1 \text { and city } 2^{[1]} \\
2 \text { physiotherapists, city } 3[3][4]\end{array}$ & $\begin{array}{l}\text { [1] At-home clinical assessments of MSD for the government } \\
\text { program }{ }^{1} \\
{ }^{[2]} \text { Evaluations of } 8 \text { clinical assessment files of MSD already } \\
\text { completed for the government program }{ }^{1} \\
\text { [3][4] Expertise with rehabilitation dogs and motor impairments with } \\
\text { children and with adults }\end{array}$ \\
\hline Dog trainers $(n=1$ female and 3 males, city 4$)$ & MSD expertise \\
\hline $\begin{array}{l}1 \text { has tetraplegia and uses a manual wheelchair; has trained dogs } \\
\text { since } 1996 .{ }^{[5]} \\
2 \text { can walk and have had trained dogs since } 1993 \text { and 2003. }{ }^{[5]} \\
1 \text { is a psychologist and has been vice-president R\&D of the dog } \\
\text { training school since } 1992 .{ }^{[5][6]}\end{array}$ & $\begin{array}{l}{ }^{[5]} \text { Experience with trained dog for people with motor impairments } \\
{[6] \text { Past experience with guide dogs for people with vision }} \\
\text { impairments }\end{array}$ \\
\hline Rehabilitation managers ( $n=1$ female and 2 males) & MSD expertise \\
\hline $\begin{array}{l}\text { Head of the mobility assistive device program for adults and seniors } \\
\text { with motor impairments, city } 1 \text { [7] } \\
\text { Director of rehabilitation technologies, city } 2^{[7]} \\
\text { Administrative director, assistive devices, city } 2^{[7]}\end{array}$ & $\begin{array}{l}{ }^{[7]} \text { Management of clinical staff in rehabilitation centers for the } \\
\text { government program }{ }^{1}\end{array}$ \\
\hline Users $(n=3$ females and 2 males, city 2$)$ & MSD expertise and main occupation \\
\hline $\begin{array}{l}\text { Non-walker, } 47 \text { years old, he has tetraplegia and uses an powered } \\
\text { wheelchair. He has severe birth defects including no upper limbs } \\
\text { and no thigh segment. He controls his wheelchair with his foot. } \\
\text { Walker and non-walker, } 39 \text { years old, she has a neurodegenerative } \\
\text { condition; she used her } 1^{\text {st }} \text { dog to walk and has had a } 2^{\text {nd }} \text { since } \\
\text { using a manual wheelchair. She has Friedreich's Ataxia. }\end{array}$ & $\begin{array}{l}{[8] \text { He has had his } 1^{\text {st }} \text { dog for } 6 \text { years. He is working. }} \\
{ }^{[9]} \text { She had her } 1^{\text {st }} \text { dog for } 3 \text { years and has had her } 2^{\text {nd }} \text { dog for } \\
3 \text { years now. She is working. } \\
{ }^{[10]} \text { He had his } 1^{\text {st }} \text { dog for } 9 \text { years and has had his } 2^{\text {nd }} \text { dog for } \\
2 \text { years now. He is working. } \\
{[11] \text { She had her } 1^{\text {st }} \text { dog for } 4 \text { years and has had her } 2^{\text {nd }} \text { dog for }}\end{array}$ \\
\hline $\begin{array}{l}\text { Non-walker, } 35 \text { years old, he has paraplegia and uses a manual } \\
\text { wheelchair. He has incomplete C5-C6 Brown-Séquard } \\
\text { tetraplegia. }{ }^{[10]}\end{array}$ & $\begin{array}{l}5 \text { years now. She is not working and has few activities outside the } \\
\text { home. } \\
{ }^{[12]} \text { She had her } 1^{\text {st }} \text { dog for } 4 \text { years, } 2^{\text {nd }} \text { dog for } 4 \text { years, and has }\end{array}$ \\
\hline $\begin{array}{l}\text { Non-walker, } 52 \text { years old, she has tetraplegia and uses a powered } \\
\text { wheelchair. She has limb-girdle muscular dystrophy. }{ }^{[11]}\end{array}$ & $\begin{array}{l}\text { had her } 3^{\text {rd }} \text { dog for } 2 \text { years now. She is active outside the home } \\
\text { but is not working any more. }\end{array}$ \\
\hline
\end{tabular}

Walker, 53 years old, she has a neurodegenerative condition and uses

the dog to walk. She has left hemiplegia following a stroke. ${ }^{[12]}$

City 1 = Quebec, City $2=$ Montreal, City $3=$ Sherbrooke, City $4=$ Sainte-Madeleine, MIRA Foundation. Note 1 : Program for the reimbursement of expenses related to the use of a mobility assistance dog, Ministère de la santé et des services sociaux du Québec [1].

\subsection{Scientific evidence supporting decision-making process when recommending a MSD}

All of the participants indicated the dog's support in helping to perform daily activities and improving satisfaction with the performance of important occupations as two essential criteria to consider in the decision to recommend a MSD or not, regardless of the user's profile (Table 2). A person with tetraplegia was the only case where improvement in psychosocial aspects was chosen among the top five criteria for recommending a MSD. For the tetraplegia profile (case A), improvement in grasping ability and the performance of daily activities obtained the highest weighted score (77/95). For the paraplegia profile (case B), help with pulling the wheelchair (82/95) and impact on reducing shoulder pain $(57 / 95)$ were the two most important criteria to include when making the decision to recommend a MSD. Finally, for people who can walk and have an incomplete spinal cord injury or degenerative dis- ease (case C), help with walking (71/95), with transfers (51/95) and daily activities (49/95) were ranked first, second and third respectively when making the decision to recommend a MSD.

\subsection{Added value of the MSD compared to other assistive devices}

Experts reported divergent opinions about the added value of a MSD compared to other assistive devices expected to be recommended typically in a similar situation in clinical practice. If a MSD was recommended, the potential added value across the three clinical cases investigated differed (Fig. 1). For people with tetraplegia (case A), the MSD is reported to be superior to the long-handled grabber, mouth stick and call button. The MSD is reported to be complementary to the door opener (the MSD opens some doors before the wheelchair gets there) and to human help for carrying small objects (the dog can fetch things and carry 
Table 2

Top five criteria or scientific evidence cited to recommend mobility service dogs (MSD) for three clinical mobility cases $(n=19)$

\begin{tabular}{|c|c|c|}
\hline Clinical mobility cases & Total score $/ 95$ & Most frequent ranking $(\max 19)$ \\
\hline \multicolumn{3}{|l|}{$\begin{array}{l}\text { A-People with tetraplegia using a powered wheelchair as their main mode of loco- } \\
\text { motion }\end{array}$} \\
\hline MSD improves grasping ability & 77 & 12 first choice \\
\hline MSD is used for daily activities & 77 & 14 second choice \\
\hline MSD increases satisfaction in important occupations & 40 & 9 third choice \\
\hline MSD improves reintegration into normal life & 39 & 10 fourth choice \\
\hline MSD has positive impacts on psychosocial aspects & 24 & 7 fifth choice \\
\hline \multicolumn{3}{|l|}{$\begin{array}{l}\text { B-People with paraplegia or a complete spinal cord injury using a manual wheelchair } \\
\text { as their main mode of locomotion }\end{array}$} \\
\hline MSD improves manual wheelchair propulsion & 82 & 13 first choice \\
\hline MSD decreases shoulder pain in manual wheelchair users with spinal cord injury & 57 & 7 second choice \\
\hline MSD is used for daily activities & 43 & 5 third choice \\
\hline MSD increases satisfaction in important occupations & 31 & 6 fifth choice \\
\hline MSD improves reintegration into normal life & 28 & 7 fourth choice \\
\hline \multicolumn{3}{|l|}{ C-People with an incomplete spinal cord injury or degenerative disease who can walk } \\
\hline MSD improves walking & 71 & 14 first choice \\
\hline MSD improves transfers & 51 & 8 second choice \\
\hline MSD helps with daily activities & 49 & 8 fourth choice \\
\hline MSD increases satisfaction in important occupations & 31 & 6 third choice \\
\hline MSD decreases shoulder pain ${ }^{1}$ & 27 & 4 first choice \\
\hline MSD improves grasping ability & 27 & 4 second choice \\
\hline
\end{tabular}

Note 1. During the focus groups, some experts mentioned that after receiving a MSD, clients' shoulder pain decreased, probably because they no longer had to use or carry another type of assistive device or because they used their upper limbs less to reach or grasp objects.

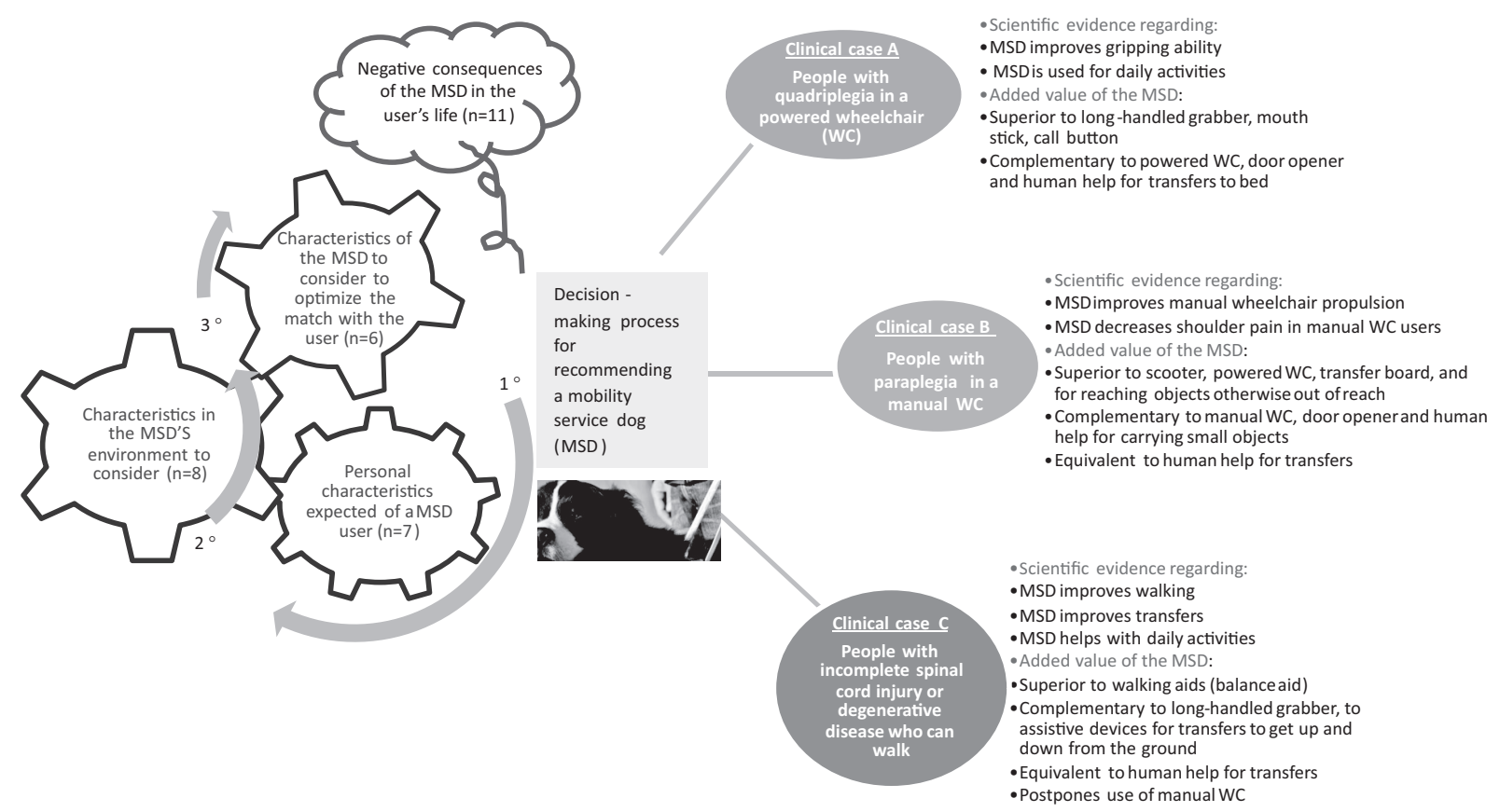

Fig. 1. Decision-making process diagram to recommend a mobility service dog.

things on its back). For people with paraplegia using a manual wheelchair (case B), the MSD was considered superior to powered mobility aids (scooter, powered wheelchair) over long distances because of its polyvalence (ability to pull on all surfaces, indoor and outdoor) and size (smaller, lighter) and to a transfer board. The MSD helps the user reach objects that are out of reach when the person is alone, which no other assistive device can do. The MSD is complementary to manual wheelchairs because it can provide traction 
aid during wheelchair propulsion, enabling the user to go faster and farther with less fatigue and effort. Finally, for people with a neurological impairment who can walk (case C), the MSD is reported to be superior to habitual walking aids for ambulation and dynamic balance control in unforeseen situations (e.g., user teetering). The MSD was identified as complementary to the long-handled grabber, especially because the user does not have to bend down to pick up things from the ground, which minimizes the risk of losing balance, or even falling. It is also complementary to other transfer devices, which help users to get up and down from the ground. The dog can approach the person to help $\mathrm{him} / \mathrm{her}$ stand up or get down on the ground, anywhere, anytime, which is not the case with transfer boards, lifts, poles and grab bars, which are static and usually located in one place. MSD enable users to transfer alone. Also, MSD trained to help with walking may postpone use of a manual wheelchair.

Please note that since the question in the focus group about the added value was "do you consider the service dog superior, inferior or equivalent to another assistive device, and justify", and since an assistive device should compensate functional aspects, this can explain why there is no mention of the psycho-social effect in the added value, like it mentioned in Table 2 for evidence supporting clinical decision making. The dog was not compared to a companion or a human being but to an object or a tool.

\subsection{Person, environment and technology matching process}

To envision partnering with a MSD, the experts said the dog's skills should be considered only after ensuring that the user has a personal profile and an environment receptive to a dog. For that receptive personal profile, seven personal characteristics expected for a future MSD user have emerged from the discussions (Table 3 ). These characteristics include attitudes (already likes or respects dogs), aptitudes (is able to care for the dog at all times: exercise, hygiene, nutrition, health; feels confident that, with the dog, can safely go out anywhere alone), behaviors (is assiduous in maintaining the dog's assistive functions; is directive and authoritative enough to make the dog obey commands) and motor skills (uses the dog to combat fatigability and shoulder pain caused when performing some daily activities; by using the MSD, wants to keep active and/or be more independent). For that receptive environment, the experts identified four level of environment to consider for recommending a MSD, namely the human (caregiver, household members), built (client's dwelling), social (neighbours, community, work or school) and organizational environment (public funding for assistive devices, program for reimbursing expenses related to looking after a MSD, housing regulations). And for the characteristics of the dog expected as a canine assistive device, the experts propose that MSD must be able to perform its assistive functions effectively, efficiently, safely and satisfactorily in the environment of each potential user. Six characteristics are cited as advantages expected of a MSD in relation to the following aspects: companion, assistive functions, focus on the task, physical strength, team work, social acceptance of the dog.

\subsection{Potential negative consequences in a MSD user's life}

Table 4 presents sentences reflecting potential negative consequences that could be hard to manage and should be discussed with clients before they get a MSD. These sentences concern stigmatization (social aspect of the dog attracting people), resilience (e.g. grieving, having to change MSD), care burden (e.g. get exercise outside every day, house cleanliness and housework, caring for the dog, regular trips to the vet, etc), authority or obedience (e.g. defining and respecting MSD rules of conduct with the dog, control over the dog's occasional unpredictable behavior, or in dangerous situations).

Figure 1 presents main elements contributing to the decision-making process for recommending a MSD, which vary across the clinical cases (right side of the diagram), although some of the results point to a few common factors across all cases (left side of the diagram). Indeed, the "scientific evidence cited" and "added value of the MSD compared to other assistive devices" do not have the same influence on users with tetraplegia, paraplegia or an incomplete injury who can walk (Fig. 1). On the other hand, many facilitating characteristics related to the individual, the environment and the dog as well as some probable negative consequences in the life of a MSD user were identified as important to consider when making the decision to recommend a MSD, regardless of the user's profile (Fig. 1). Specific results have been presented above in light of the research questions. 
Table 3

Personal, environmental and canine characteristics to consider in the decision-making process for recommending mobility service dogs (MSD)

$1^{\text {st }}-$ Personal characteristics expected of a MSD user

1. Already likes or respects dogs /A ttitude

2. Is able to care for the dog at all times (exercise, hygiene, nutrition, health)/A ${ }^{\text {ptitude }}$

3. Is assiduous in maintaining the dog's assistive functions/B $\mathbf{B}^{\text {ehavior }}$

4. Feels confident that, with the dog, can safely go out anywhere alone /B ehavior

5. Is directive and authoritative enough to make the dog obey commands / $\mathrm{B}$ ehavior

6. Uses the dog to combat fatigability and shoulder pain caused when performing some daily activities /M otorskills

7. By using the mobility service dog, wants to keep active and be more independent / $\mathrm{M}^{\text {otorskills }}$

$2^{\text {nd }}-$ Characteristics in the MSD's environment to consider

1. Caregiver $/ \mathrm{H}^{1}$ : Does the dog live under the same roof as the caregiver? Is the dog a burden or does it give the caregiver some respite? Who has to care for the dog (e.g., clean its ears)?

2. Household members $/ \mathrm{H}^{1}$ : Do family members like dogs? Do they have any allergies? Who feeds the dog and takes it for a walk? Who cleans up after the dog and vacuums up the hair? Are there any other animals in the home who may interfere with controlling the dog? Is the dog a burden for family members?

3. User's dwelling/ $\mathbf{B}^{1}$ : Is it clean and safe and welcoming for the dog? Does the environment seem dysfunctional (e.g., squalid, overcrowded, dog malnourished)?

4. User's general environment $/ \mathrm{S}^{1}$ : Can the dog user get help to care for the dog in the apartment/residence, building where they live, in the community, at work or at school?

5. School/ $/ \mathrm{S}^{1}$ : If children make a fuss of the dog, does it interfere with controlling the $\operatorname{dog}$ ?

6. Public funding for assistive devices $/ \mathrm{O}^{1}$ : When the mobility assistive device is chosen (walker, manual/powered wheelchair, scooter), is the MSD the best option for the client to access more places and more objects?

7. Program for reimbursing expenses related to looking after a MSD $/ \mathrm{O}^{1}$ : If the MSD can no longer perform its assistive functions (sick, injured, deconditioned) but the dog's master wants to keep it anyway, is the client financially able to cover the costs of looking after the $\operatorname{dog}$ ?

8. Housing regulations $/ \mathrm{O}^{1}$ : Is having a dog allowed? Is it worth moving in order to be able to have a dog? Is it worth advocating for the client's rights (long process, risk of stigmatizing the client)?

$3^{\text {rd }}$ - Characteristics of the dog expected as a canine assistive device

1. The dog is a good companion: Will the MSD be used in many daily activities? Will the dog travel a lot? Can the MSD always follow its master? Is the dog continent when travelling?

2. The dog safely performs the assistive functions taught during training.

3. The dog is not distracted in an enticing environment: it does not gravitate towards other animals or food.

4. The dog is not overwhelmed physically: What weight or load can its skeleton bear? For example, a 300-lb load on the dog's back during transfers, several times a day, probably represents a risk to the dog's health. What effort can the dog make based on its cardio-respiratory capacity? For example, a 40-km run with the MSD or going up several steep hills on a daily basis probably represents a risk to the dog's health.

5. The dog works well with its master, the dog's and user's personalities are well matched.

6. The breed of dog has a good reputation in the community.

Note 1. Environment level: $\mathrm{H}=$ human, $\mathrm{B}=$ built, $\mathrm{S}=$ social, $\mathrm{O}=$ organizational.

\section{Discussion}

This study provides concrete guidelines to support the decision-making process of rehabilitation professionals involved in recommending MSD, or at least a first consensus around three clinical cases. According to our results, for MSD recommendations to be effective, one must first recognize that the reasons for recommending a MSD can vary based on the clinical situation. A person with tetraplegia using a powered wheelchair was the only case where improving the psychosocial aspect was listed among the top five criteria for recommending a MSD. Our results illustrated that the added values of the MSD compared to other assistive devices are different for each clinical case. However, regardless of the clinical profile, it is important to consider the user's personal and environmental charac- teristics before envisioning recommending a MSD for its assistive functions. Also, in this process of matching person and technology, it is essential that users and their families are informed of the potentially negative consequences of using a MSD.

The results of this study with respect to the "added value of MSD versus other assistive devices" are interesting, especially in today's context where rehabilitation professionals need guidelines to warrant recommending a MSD over traditional assistive devices, that may be easier to obtain and at a lesser cost. Rehabilitation professionals must be encouraged not to focus on technology in its broad sense (assistive devices and MSD). Before suggesting that a MSD could be superior, equivalent or complementary to traditional assistive devices, they need to carefully document or assess the characteristics and receptiveness of the poten- 
Table 4

Potential negative consequences in the life of a mobility service dog (MSD) user

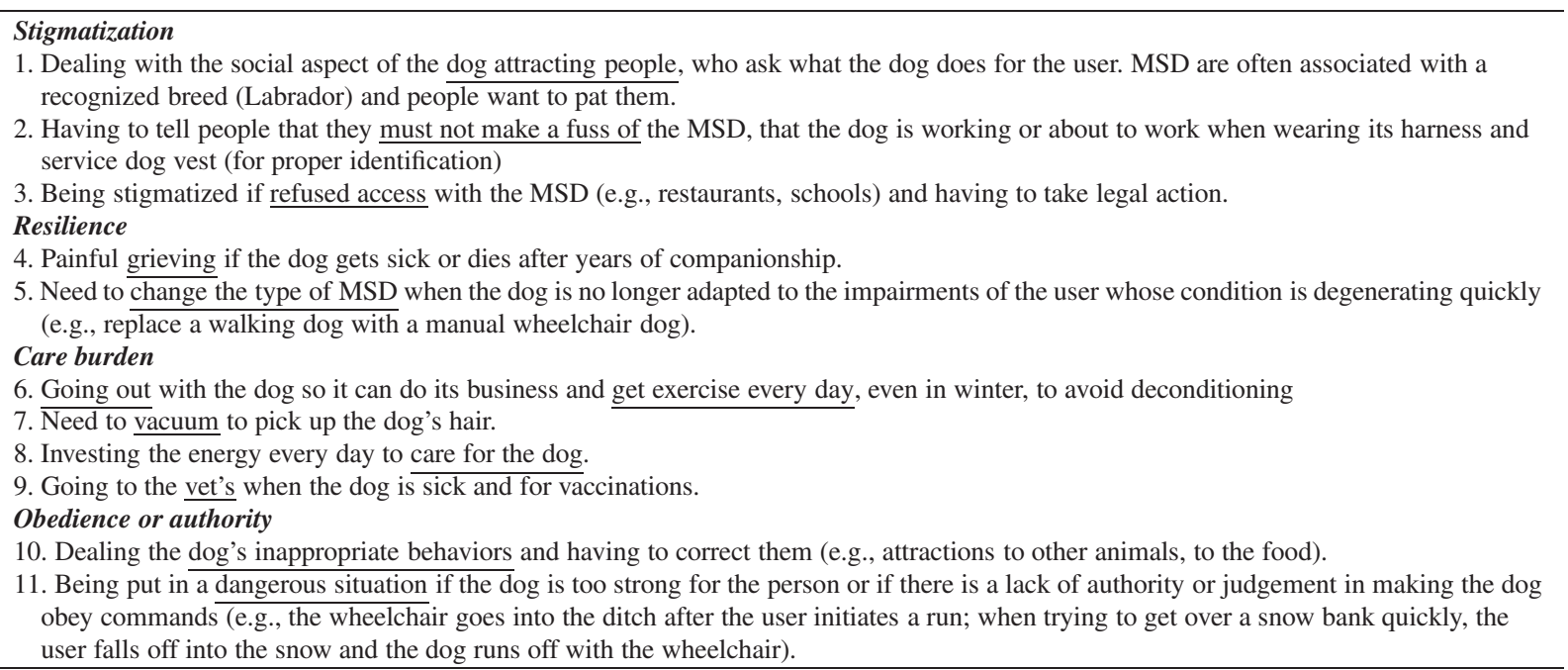

tial user and the environment. Subsequently, this assessment must be matched to the users' profile as our study revealed that perceived added value of using a MSD and perceived strength of the evidence currently available to support the decision-making process are different for users of powered or manual wheelchairs or walkers.

\subsection{Challenges for rehabilitation professionals}

Elements of the person, the environment and the dog and their interactions are an integral part of the process of recommending a MSD. The challenge for health professionals is to know how to measure these elements and how they interact, especially as some of these aspects can change over time. In this context, a recent article recommending the use of the Impact on Participation and Autonomy (IPA) scale as an important first step for Mobility Dogs ${ }^{\circledR}$ to test the benefits of trained service dogs may warrant consideration [17]. However, a serious limitation of the IPA is its focus on benefits rather than determinants and predictors that influence the recommendation of a MSD. Unlike conventional mobility aids (e.g., cane, wheelchair) which can be loaned to and tested with the user to evaluate the potential benefits for that particular person, loaning a MSD is logistically complex and is, first and foremost, a living assistant 'customized' to the user's needs. In fact, matching a specific mobility service $\operatorname{dog}$ and a unique user typically requires many days of training and sufficient time for the new dog-user duo formed to become acquainted with each other. A potential solution to better inform the decisional process leading to the recommendation a service dog may be to plan a trial period with a 'rehabilitation dog' to explore its potential usability. A 'rehabilitation dog' is typically owned by a rehabilitation facility and has been trained to accommodate the needs of multiple users when performing a wide range of functional tasks typically trained during functional rehabilitation [18]. Moreover, such a trial, may also allow one to pinpoint the potential future user attitudes and aptitudes towards the mobility service dog and, to a lesser extent, the environment's receptiveness to it. This first step could be followed by a visit to the home or school with a rehabilitation dog to see if the person's household members and dwelling are receptive. Finally, to simulate and inform potential users of the possible negative consequences of a MSD in a user's life, a short video could be made to inform them about their various responsibilities with respect to the dog itself, which means not only taking care of the dog but also maintaining its conditioning for its assistive functions. For example, this involves taking the dog out in inclement weather to keep in conditioned MSD and having to do the extra housework that comes with having a dog in the home, as well as coping with a MSD that is sick, dies or has to be replaced if it can no longer perform the assistive functions originally planned. The video, coupled with the experience with the rehabilitation dog, should provide a reasonably accurate and informative simulation to support the rehabilitation professionals' decision regarding whether or not to recommend a MSD. 


\subsection{Study strengths and limitations}

This study has both strengths and limitations. Based on the four criteria for scientific rigor used to judge the quality of qualitative research [19], that are here presented in bold in text, we judge that the study's truth value is acceptable. In other words, our study provides a credible representation of the phenomenon being studied and one that is probably quite comprehensive, by displaying four distinct research questions, three clinical cases, various types of expertise encompassing the MSD recommendation process and adapted interview technique.

Concerning the study's applicability, we judge that the results can be generalized to contexts similar to the three clinical cases A, B and C (relatively similar issues or concepts raised through the focus groups). We also provide a dense description of experts in Table 1 as a transferability strategy, as recommanded by Krefting [19]. Response bias is possible in the context of the present study. First, all MSD users who accepted to participate in the focus group were possibly enthusiastic regarding their past personal experience with their MSD and may have provided desirable responses to avoid jeopardizing indirect benefits linked to the MSD (e.g., monthly financial allowance for the MSD via a publicly-funded program in Québec). The results on "added value of MSD", as an example, would have been different if one or more MSD unsatisfied user(s) had been invited to join the discussion group. Second, all experts who accepted to participate in the focus group are currently or were in the recent past full or part-time employees of the publiclyfunded MSD program or supported financially by other sources and, as a consequence, were actively involved in the decision-making process for recommending a MSD or in training and proving the MSD. Hence, they may have aligned there answer with what is expected in terms of positive outcomes and measurable from the program in itself and provided overly desirable responses with respect to the usability of the MSD when compared to other stakeholders. Other stakeholders, like merchants who must accommodate users of MSD (e.g. restaurant, banks and shops), funders of the health system, administrators of other assistive devices programs or economists interested in health and welfare programs, may have brought different perspective to the discussions.

With respect to the study's consistency, we judge that the reproducibility of the methods is optimal and a strength of the study. In fact, most methodological de- cisions are auditable (interview guide validated by six researchers, 10 research statements about the efficacy of the MSD, definition of the variables/themes used before coding, diagram used for categorizing themes and deductible themes, peer examination process).

Concerning the study's neutrality, only some themes remained equivocal even after validation between the researchers, but we feel this inconsistency can be explained for the most part. For example, it is understandable that the sub-themes "The MSD is complementary to human help" and "The MSD is equivalent to human help" could overlap since there was no consensus in the discussion concerning this. Sometimes the dog helps one caregiver to get a client into bed instead of having two caregivers to do it (complementary); and sometimes the MSD replaces totally the caregiver for the transfer to bed (equivalent to human help). Also, when one says that "the MSD is superior or complementary to a call system", sometimes the MSD may in fact replace it and do a better job, but sometimes the dog is an addition to other tools already owned by the client. The "added valued of MSD" themes are more linked to the verbatim extracted from discussion transcripts than other themes presented in the diagram for decision-making process for recommending a mobility service dog. Also, we used as a confirmability strategy, reflexivity of OT and PT coresearchers to comment on the content of the tables of results.

Further studies are needed to investigate other clinical situations/clinical vignettes in which the use of a MSD could be considered. We think the participation of individuals with other diagnoses and different ages in a rural setting, or with different requirements surrounding the use of a MSD and traditional assistive devices (e.g., 20-year-old quadruple amputee who plays sport and lives in a vacation area) might have enriched the discussions and possibly brought up additional viewpoints.

\section{Conclusions}

This study allowed us to formulate general guidelines to support the decision-making process of rehabilitation professionals involved in recommending MSD for people with tetraplegia using a powered wheelchair as their main mode of locomotion, with paraplegia using a manual wheelchair, or with an incomplete spinal cord injury or degenerative disease who can walk. Through focus groups with 19 experts 
investigating the process of recommending a MSD (occupational therapists, physiotherapists, MSD trainers, managers of the program for reimbursing expenses related to the MSD, and MSD users), important information was gathered. Part of this information was systematized in a decision-making process diagram for recommending a mobility service dog, displaying six main variables: scientific evidence cited, added value of the MSD, personal, environmental and canine aspects to consider, and possible negative consequences of having a MSD. A consensus emerged regarding the perceived added value of the MSD compared to other assistive devices, but this also varied from one clinical case to the next. We also found a consensus regarding the priorities to consider in the decision-making process of recommending a MSD, namely, first, evaluation of the person (7 characteristics); second, receptiveness of the MSD's environment (4 contexts); and third, consideration of the advantages of a MSD (6 characteristics of the dog). Finally, a consensus emerged from our study regarding the need to better inform rehabilitation professionals and future users regarding possible negative consequences associated with the use of a MSD. The results of this study could be used as a guide for occupational therapists and physiotherapists who are considering recommending a MSD to their clients with motor impairments (similar to the clinical cases in our study) or who have been asked to evaluate the actual use of a MSD as an assistive device in a client's life.

\section{Acknowledgements}

The Assistance Dog for Mobility Impairments (ADMI) group includes six researchers in physical rehabilitation who have worked together since 2010 . Four are the authors of this paper; the others are François Routhier, Eng, PhD, and Michel Tousignant, PT, PhD. The authors would like to thank Noël Champagne, the research vice-president of Mira Foundation for the recruitment of dog trainers and his constant enthusiasm regarding research projects with the ADMI group. Thank you to Valérie Martin-Lemoyne, PT, for her assistance with recruiting dogs users for this study, and Frederic Dumont for his assistance with planning the focus groups and data analysis for Table 2. The authors also wish to thank the 19 experts who generously shared their experience during the focus groups. The study was funded by the Consortium for Research Development in Traumatology of the Fonds de recherche du Québec Santé (FRQ-S).

\section{Conflict of interest}

The authors declare they have no conflict of interest.

\section{References}

[1] MIRA Foundation [Appication form assistance dog]. Québec. Canada. [updated 2016 August 18 cited 2016 August 3] Available from: http://www.mira.ca/en/programs/7/IMG/pdf/ Application_for_an_assistance_dog_v3dec2009.pdf.

[2] Governement of Québec [homepage on the Internet]. Québec. Programme de remboursement de frais relatifs à l'utilisation d'un chien d'assistance à la motricité. [updated 2015 December 12; cited 2016 June 9] Available from: http://sante.gouv. qc.ca/programmes-et-mesures-daide/programme-deremboursement-de-frais-utilisation-chien-assistance-a-lamotricite/.

[3] Blanchet M, Gagnon DH, Vincent C, Boucher P, Routhier F, Martin-Lemoyne V. Effects of a Mobility Assistance Dog on the Performance of Functional Mobility Tests Among Ambulatory Individuals with Physical Impairments and Functional Disabilities. Assistive Technology. 2013; 25(4): 247-52.

[4] Champagne A, Gagnon DH, Vincent C and members of the ADMI group. Comparison of cardiorespiratory demand and rate of perceived exertion during propulsion in a natural environment with and without the use of a mobility assistance dog in manual wheelchair users. American Journal of Physical Medicine and Rehabilitation. 2016; 95(9): 685-91.

[5] Gagnon DH, Blanchet M, Martin-Lemoyne V, Vincent C, Routhier F, Corriveau H. Using a mobility assistance dog reduces upper limb effort during manual wheelchair ramp ascent in an individual with spinal cord injury. Journal of Spinal Cord Medicine (ISSN: 10790268). The journal of spinal cord medicine. 2015; 36(6): 700-6.

[6] Hubert G, Tousignant M, Routhier F, Corriveau H, Champagne N. Effect of service dogs on manual wheelchair users with spinal cord injury: A pilot study. Journal of Rehabilitation Research \& Development. 2013; 50(3), 341-350.

[7] Martin-Lemoyne V, Gagnon DH, Routhier F and ADMI group (Vincent C). To what extent can the use of a mobility assistance dog reduce upper limb efforts when manual wheelchair users ascend a ramp. Martin-Lemoyne V, Gagnon $\mathrm{DH}$, Routhier $\mathrm{F}$ and ADMI group (Vincent C). To what extent can the use of a mobility assistance dog reduce upper limb efforts when manual wheelchair users ascend a ramp. J Appl Biomech. 2016; 32(2): 186-95.

[8] Vincent C, Joyal I, Gagnon DH, Routhier F. Étude rétrospective transversale de l'utilisation d'un chien d'assistance à la motricité au Québec. Research report. Centre de documentation, Institut de réadaptation en déficience physique de Québec, Québec, QC, Canada, 46pp. Déposé au MSSS. 2012.

[9] Vincent C, Gagnon DH, Routhier F, Leblond J, Boucher P, Blanchet M, Martin-Lemoyne V. Service dogs in the province of Quebec: sociodemographic profile of users and the dogs impact on functional ability. Disability and rehabilitation: Assistive technology. 2015; 10(2): 132-40.

[10] Vincent C, Gagnon DH, Routhier F, Dumont F, Poissant L, Corriveau H, Tousignant M. Service Dogs for People with Spinal Cord Injury: Outcomes Regarding Functional Mobility and Important Occupations. Stud Health Technol Inform. 2015; 217: 847-51. 
[11] Fine AH. Handbook on animal - assisted therapy. Theoritical fondations and guidelins for practice. Third edition. Amsterdam: Elsevier. 588 pp. 2010.

[12] Grudin J. Utility and usability: Research issues and development contexts. Interacting with Computers. 1992; 4(2), 209217.

[13] Mayhew D. The usability engineering lifecycle: A practitioner's handbook for user interface design. Morgan Kaufmann Publishers, San Francisco, 1999.

[14] Scherer M. [homepage on the Internet]. Matching Person and Technology (MPT). Assessment Process. updated 2016 February 13; cited 2016 June 9]. Available from: http://www. matchingpersonandtechnology.com/mptdesc.html.

[15] Morgan, D.L. The focus group kit - Planning Focus Groups. Thousand Oaks, CA: Sage Publications. 1998.
[16] Braun V, Clarke V. Using thematic analysis in psychology. Qual Res Psychol. 2006; 3: 77-101.

[17] Mudge S, Rewi D, Channon A. Identifying an outcome measure to assess the impact of Mobility Dogs. Disability and Rehabilitation: Assistive Technology. 2015. Available from DOI: 10.3109/17483107.2015.1079267.

[18] Rondeau L, Corriveau H, Bier N, Camden C. Effectiveness of a rehabilitation dog in fostering gait retraining for adults with a recent stroke: A multiple single-case study. NeuroRehabilitation. 2010; 7(2): 155-163.

[19] Krefting L. Rigor in qualitative research: the assessment of trustworthiness. American Journal of Occupational Therapy. 1991; 45: 214-222. 